\title{
Fundamentos para la extensión de la edad pediátrica hasta el término de la adolescencia a nivel de toda la red asistencial de salud. Recomendación del Comité de Adolescencia de la Sociedad Chilena de Pediatría
}

VERÓNICA GAETE P., M. EUGENIA HENRÍQUEZ C., PAZ ROBLEDO H., TAMARA ZUBAREW G., ELDRETH PERALTA V., CLAUDIA SAGREDO B., FRANCISCO FUNES D., COMITÉ DE ADOLESCENCIA, SOCIEDAD CHILENA DE PEDIATRÍA

\section{Definición del problema}

El sistema de salud chileno no satisface adecuadamente gran parte de las necesidades de salud del grupo adolescente, a excepción de las del ámbito reproductivo ${ }^{1-3}$. $\mathrm{Al}$ menos parcialmente, ello se debe a una estructuración inadecuada de la atención, que escinde al grupo juvenil, especialmente a nivel de la Atención Secundaria y Terciaria (mayores y menores de 15 años). Esto ha generado:

- Una ausencia de visión de los adolescentes como tales, quienes han sido erróneamente asumidos como otros niños más en el sistema pediátrico y como adultos en la red de atención de la adultez. Se les ha abordado sin una perspectiva del desarrollo propio de esta etapa de la vida, que además sea continua y progresiva, dificultando su proceso de crecimiento y desarrollo psicosocial, y finalmente el logro de su autonomía y autocuidado en salud 4 . Por lo mismo, ha favorecido la pérdida de oportunidades de promoción y prevención, con el consiguiente aumento de la carga de enfermedad ${ }^{5}$.

- Que se hayan hecho invisibles las necesida- des de salud de este grupo de edad, que son específicas $^{5-14}$, debido también al fenómeno previo (no visualización de los adolescentes como tales por la infantilización de los menores de 15 y la percepción de los mayores como adultos). Ello ha dificultado el abordaje de su problemática, en especial el de las conductas de riesgo, los problemas de salud mental y los conflictos éticos asociados a su atención ${ }^{15,16}$. Además y por lo mismo, el personal de salud no ha recibido una capacitación adecuada en el manejo de los adolescentes, lo que ha perpetuado los problemas y favorecido la iatrogenia ${ }^{4,17}$.

- Un enfrentamiento inadecuado de los jóvenes con patología crónica y sus familias, a quienes se les ha interrumpido la atención en la mitad de un período muy vulnerable de la vida, lo que con frecuencia ha generado crisis que han llevado, entre otras cosas, a la pérdida de tratamientos y, posiblemente, hasta de vidas. La situación se ha visto agravada en la práctica, por las consecuencias administrativas y de salud producto de la tendencia a la retención de estos adolescentes en el sistema pediátrico. El impacto 
de todo esto resulta cada vez mayor debido al aumento progresivo de las enfermedades crónicas propio de la situación epidemiológica actual.

- Un incremento de los costos de atención de este grupo de edad por los problemas previamente planteados, que han conducido hasta ahora a un aumento de la carga de enfermedad, a un déficit de adherencia y/o fracaso de tratamientos, a la retención de los crónicos en los hospitales pediátricos, etc.

Lo anteriormente detallado hace evidente que los límites actuales de la atención pediátrica y del adulto han llevado a un abordaje de salud deficiente de los adolescentes chilenos y sus familias, generando problemas tanto en su acceso a servicios adecuados, como en la continuidad y pertinencia de su atención de salud, e incrementando los costos de la misma.

\section{Alternativas de solución}

Se visualizan dos alternativas de solución para la problemática previa:

- Establecer un sistema de atención destinado exclusivamente a los adolescentes, con dependencias específicas en las que se resuelvan las necesidades clínicas de todo este grupo de edad. Este modelo puede funcionar cuando el nivel de ingresos de la población es alto, situación que dista mucho de la de nuestro país.

- Prolongar la edad pediátrica hasta el término de la adolescencia a nivel de toda la red asistencial de salud, de manera de que la demanda clínica del grupo adolescente completo sea resuelta dentro de un sistema de atención infanto-juvenil adecuadamente capacitado y acondicionado.

\section{Antecedentes que avalan la extensión de la edad pediátrica hasta el término de la adolescencia como mejor alternativa de solución a nivel nacional}

\section{Necesidades de atención de salud de los adolescentes}

Sin duda es el equipo de salud pediátrico, adecuadamente capacitado, el que posee las condiciones para brindar una atención de las características necesarias para esta etapa del ciclo vital. Éstas son, entre otras:

\section{Atención Integral}

Requiere de un enfoque biopsicosocial, preventivo y de desarrollo $o^{3,6,18-21}$. Ello, porque en la adolescencia muchos de los problemas de salud poseen una naturaleza biopsicosocial e incluso las enfermedades tradicionalmente biomédicas repercuten con frecuencia en los ámbitos psicológico y social ${ }^{6,22-24}$. Por otra parte, debido a que una proporción importante de la problemática juvenil resulta prevenible $e^{6,25-27}$ -en especial las conductas de riesgo y sus consecuencias- y frecuentemente no constituye motivo de consulta para el adolescente y/o su familia hasta que es ya de difícil resolución ${ }^{28,29}$. Por último, porque se trata de un período en el cual se producen múltiples y rápidos cambios biológicos, psicológicos y sociales que condicionan tanto vulnerabilidades como oportunidades para los jóvenes, quienes requieren de una orientación adecuada para alcanzar un desarrollo positivo ${ }^{18,30-32}$.

\section{Abordaje familiar}

La adolescencia es la etapa de la vida en que se transita progresivamente desde la absoluta dependencia en los padres hacia -potencialmente y dependiendo de las condicionesla total autonomía. Durante todo este período los padres son habitualmente el principal sistema de apoyo del joven y fundamentales para su adecuada atención de salud y cumplimiento de las indicaciones ${ }^{33-36}$. Por otro lado, la familia también constituye la génesis de parte de la problemática adolescente ${ }^{37-41}$. Así, resulta esencial involucrarla en el abordaje de salud del joven.

\section{Continuidad de la atención y adecuada relación médico-paciente}

Separar al joven de su equipo de atención antes de que haya completado su proceso de adolescencia -sobre todo si padece de una enfermedad crónica-, provocará muchas veces un quiebre en su relación con el sistema de salud y su mala adherencia a las indicaciones y los controles. La transición en el cuidado de 
salud debe ser un proceso gradual e, idealmente, la transferencia al sistema de atención del adulto tiene que producirse una vez terminado el crecimiento y desarrollo del joven, cuando éste ya posee capacidad de cuidar de su propia salud y de tomar decisiones autónomas en este ámbito ${ }^{18,42,43}$.

Es el equipo pediátrico el que tiene una formación profesional que le permite una visión evolutiva del crecimiento y desarrollo, un enfoque preventivo en salud y relacionarse adecuadamente tanto con los adolescentes como con sus familias. Además, se encuentra en una posición de privilegio para dar continuidad a la atención del joven, ya que, producto de haberla llevado a cabo desde la más temprana infancia, conoce en profundidad al adolescente y sus padres, y ha establecido un vínculo de confianza con ambos.

\section{La experiencia extranjera}

\section{Posición de sociedades científicas internacionales}

La Academia Americana de Pediatría ha explicitado y reafirmado que la responsabilidad de la pediatría llega habitualmente hasta los 21 años de edad ${ }^{44-47}$.

\section{Atención del adolescente a nivel internacional}

Grandes hospitales y universidades de EE.UU., Australia y Europa (Francia, España e Inglaterra) han incorporado progresivamente la atención del adolescente a sus servicios pediátricos, estableciendo los 18-21 años como la edad de transferencia a los servicios de adultos. Ejemplos en EEUU, entre muchos otros, son el Boston Medical Center ${ }^{48}$, el Children's National Medical Center de Washington DC ${ }^{49}$, el Children's Hospital de Los Angeles ${ }^{50}$, el Children's Hospital de Miami ${ }^{51}$ y las escuelas de medicina de la Universidad de Minnesota ${ }^{52}$, Universidad de California, San Francisco ${ }^{53}$ y Universidad de Stanford ${ }^{54}$. Lo mismo ha empezado a suceder en el contexto latinoamericano. Cuba extendió ya la atención pediátrica hasta los 17 años, 11 meses y 29 días ${ }^{55}$ y Brasil la prolongó hasta los 18-21 años en sus principales hospitales infantiles ${ }^{56}$.

\section{La experiencia nacional}

A pesar de la ausencia de una determinación política de ampliar la edad de atención pediátrica hasta el término de la adolescencia por parte de la autoridad sanitaria hasta ahora, diversas instituciones públicas y privadas de salud ya se han ido haciendo cargo de esta necesidad emergente e impostergable.

En las últimas décadas, la atención diferencial de jóvenes se ha extendido en los servicios pediátricos de nuestro país. Los Departamentos de Pediatría de la Universidad de Chile y P. Universidad Católica cuentan con centros docente-asistenciales de atención ambulatoria para adolescentes liderados por pediatras especialistas en adolescencia. La P. Universidad Católica estableció además la atención pediátrica hasta los 18 años en la mayor parte de la Red Salud UC. A nivel del sistema público de salud también se han ido desarrollando espacios para la atención de jóvenes ligados a los servicios pediátricos. Ejemplos de ello son las unidades de adolescencia de los hospitales Roberto del Río, Sótero del Río y Padre Hurtado, y el proyecto de ampliación de la edad de atención hasta fines de la adolescencia en que se encuentra trabajando el Hospital Luis Calvo Mackenna. El sistema privado también se ha hecho eco de esta necesidad. Es así como grandes clínicas han ido incorporando especialistas y/o profesionales capacitados en el área, y desarrollando centros destinados exclusivamente a la atención de este grupo de edad dependientes de sus departamentos de pediatría, como la Unidad de Adolescencia de Clínica Sta. María y el Centro de Adolescentes y Jóvenes de Clínica Las Condes.

\section{Costos asociados}

Los significativos cambios que el envejecimiento poblacional ha introducido los últimos años en la demanda de atención de salud, en especial a nivel hospitalario, hacen ventajoso que a partir de ahora sean los hospitales pediátricos los que se hagan cargo de la atención de todo el grupo adolescente. Por una parte, la disminución de la población infantil ha traído consigo una caída progresiva en las tasas de ocupación de camas en los hospitales pediátricos, y por otra, la demanda de salud asociada 
a la creciente sobrevida de los adultos mayores ha comenzado a saturar aquellos recintos destinados a la población mayor de 15 años ${ }^{57}$. En este escenario, el que sea la red pediátrica la que en adelante atienda a todo el grupo adolescente permitiría gestionar mejor los recursos, pues facilitaría la descompresión de los hospitales de adultos por una parte, y por otra, optimizar la utilización futura de la infraestructura, equipamiento y personal actualmente existentes en la red infantil a través de su redestinación hacia la atención de la población infanto-juvenil.

\section{Capacidad de capacitación en adolescencia para profesionales de la salud a nivel del país}

En Chile, las instancias de formación de calidad en salud del adolescente se han desarrollado predominantemente ligadas al ámbito pediátrico y a otras especialidades de atención infanto-juvenil (psiquiatría y ginecología), siendo las primeras aquellas que se hacen mayoritariamente cargo de la docencia en Medicina del Adolescente. Los Departamentos de Pediatría de la Universidad de Chile y la División de Pediatría de la Pontificia Universidad Católica han integrado la adolescencia dentro del currículum de formación de los pediatras y ofrecen también un diplomado en salud integral del adolescente vía internet dirigido a los diversos integrantes del equipo de atención de este grupo de edad ${ }^{58,59}$. La Facultad de Medicina de la Universidad de Chile brinda además la subespecialidad de Adolescencia destinada a pediatras ${ }^{60}$. Estos programas han permitido capacitar a un núcleo de profesionales del área pediátrica que está realizando atención diferencial de jóvenes en las instancias antes señaladas, y poseen bastante capacidad para absorber la demanda de capacitación que genere una eventual extensión de la edad pediátrica en toda la red de atención de salud (en especial los diplomados vía internet).

\section{Límites de edad del período adolescente}

La adolescencia es aquella etapa del desarrollo ubicada entre la infancia y la adultez, en la que ocurre un proceso creciente de madura- ción física, psicológica y social del ser humano, que lo lleva a transformarse en un adulto. Su inicio se asocia generalmente a fenómenos biológicos (pubertad) y su término a hitos psicosociales (adquisición de la autonomía y consolidación de la identidad).

Es escaso el consenso en relación a sus límites de edad ${ }^{61,62}$. Ello, entre otras cosas, porque existe gran variabilidad individual en cuanto a las edades en que los fenómenos que condicionan su principio y final se producen, más aún en la sociedad moderna, donde el retraso de la madurez psicológica y social ha sido progresivo ${ }^{18,63}$.

La definición operativa hasta ahora más utilizada en el ámbito de Chile y Latinoamérica es la de la Organización Mundial de la Salud (OMS) y la Organización Panamericana de la Salud (OPS), que consideran adolescencia al período comprendido entre los 10 y 19 años de edad ${ }^{62}$.

\section{Recomendación del Comité de Adolescencia de la Sociedad Chilena de Pediatría}

En base a todo lo previamente señalado, el Comité de Adolescencia de la Sociedad Chilena de Pediatría recomienda categóricamente la extensión de la edad pediátrica hasta el término de la adolescencia a nivel de toda la red de atención de salud, considerando como límite de edad aquel establecido por OMS/OPS.

\section{Nota en relación a la terminología:}

A lo largo de este documento los términos 'adolescente(s)' y 'joven(es)' son utilizados indistintamente para referirse al mismo grupo de edad. Así también, toda vez que se hace mención a 'el' o 'los' adolescente(s) o joven(es), se está aludiendo a personas de ambos sexos de estas edades. Por último, bajo la denominación 'padre(s)' se incluye a la(s) madre(s) y a otros adultos que ejerzan el rol parental.

\section{Referencias}

1.- Ministerio de Salud, UNFPA CHI1R11A, FLACSOChile: Diagnóstico de la situación del embarazo en la adolescencia en Chile, 2008. 
2.- Dides C, Benavente C, Pérez S, Guajardo A, Morán J: Estudio sobre percepciones, actitudes, significaciones, opiniones, prácticas y demandas de adolescentes y jóvenes sobre salud integral, con énfasis en la salud sexual y reproductiva. FLACSO Chile, UNFPA, Ministerio de Salud, 2007.

3.- Robledo P, Baeza J: Política nacional de salud de adolescentes y jóvenes. Ministerio de Salud de Chile 2008-2015. http://www.codajic.org/node/29, última visita 24-04-2011.

4.- Hoffmeister L: Diagnóstico de salud -incluida salud sexual y reproductiva e ITS/VIH/SIDA- de adolescentes y jóvenes de Chile. Programa de Salud de Adolescentes, Ministerio de Salud, 2008.

5.- UNICEF: The state of the world's children 2011: Adolescence an age of opportunity, 2011.

6.- Gaete V: Atención clínica del adolescente que consulta por un problema de salud. Rev Med Clin Condes 2011; 22 (1): 5-13.

7.- Gobierno de Chile: Octavo Estudio Nacional de Drogas en Población Escolar de Chile, 2009. $8^{\circ}$ básico a $4^{\circ}$ medio. Resultados principales, 2010.

8.- Instituto Nacional de la Juventud: Sexta Encuesta Nacional de Juventud. Principales resultados, 2009.

9.- De la Barra F: Epidemiología de trastornos psiquiátricos en niños y adolescentes: Estudios de prevalencia. Rev Chil Neuro-Psiquiatr 2009; 47 (4): 303-14.

10.- Rodríguez $L$ : Situación nutricional del escolar y adolescente en Chile. Rev Chil Pediatr 2007; 78 (5): 523-33.

11.- Romero M, Díaz F, Rubio P: Mortalidad por suicidio en adolescentes y jóvenes 1983-2003. Rev Chil Pediatr 2006; 77(6):623.

12.- Pakpreo P, Klein J, Neinstein L: Vital statistics and injuries. En: Neinstein L, Gordon C, Katzman D, Rosen D, Woods E, eds. Adolescent Health Care. A Practical Guide. Fifth Edition, Philadelphia: Wolters Kluwer Health / Lippincott Williams \& Wilkins, 2008: 81-113.

13.- Hagan J, Shaw J, Duncan P, eds: Bright Futures: Guidelines for health supervision of infants, children, and adolescents, 3rd edition. Elk Grove Village, IL: American Academy of Pediatrics, 2008.

14.- Jenkins $R$ : The epidemiology of adolescent health problems. En: Kliegman R, Behrman R, Jenson H, Stanton B. Nelson textbook of pediatrics, 18th edition, Philadelphia, USA: Saunders Elsevier, 2007:813-815.

15.- Ministerio de Salud, Gobierno de Chile: Encuesta Nacional de Salud ENS 2009-2010.

16.- Ministerio de Salud, Organización Mundial de la Salud, Organización Panamericana de la Salud y Centros para Control y Prevención de Enfermedades de Atlanta,
EEUU: Encuesta Mundial de Salud Escolar, Chile 2004.

17.- Luengo X, Tijero M, Zepeda A: El programa de salud de los y las adolescentes Chile 1995-2005. Instituto Chileno de Medicina Reproductiva, 2006.

18.- National Health Service Scotland. Better health, better care: Hospital services for young people in Scotland. The Scottish Government, Edinburgh, 2009.

19.- Ministerio de Salud, Subsecretaria de Salud Pública, División de Prevención y Control de Enfermedades, Departamento de Salud Mental, Programa de Salud del Adolescente y Joven: Orientaciones técnicas. Atención de adolescentes con problemas de salud mental. Dirigida a los equipos de Atención Primaria de Salud. 2009. http://www.redsalud.gov.cl/portal/url/item/87faeecd259 f328de04001011e010701.pdf, última visita 24-04-2011.

20.- American Academy of Pediatrics. Committee on Adolescence: Achieving quality health services for adolescents. Pediatrics 2008; 121 (6): 1263-70.

21.- McIntyre P: Adolescent friendly health services. An agenda for change. WHO/FCH/CAH/02.14, World Health Organization, 2002. http://whqlibdoc.who.int/ hq/2003/WHO_FCH_CAH_02.14.pdf, última visita 24-04-2011.

22.- Coupey M: Chronic illness in the adolescent. En: Neinsten L, Gordon C, Katzman D, Rosen D, Woods E. Adolescent Health Care. A practical guide. Fifth Edition, Philadelphia: Wolters Kluwer Health / Lippincott Williams \& Wilkins, 2008: 1057-66.

23.- Jones $R$, Bradley $E$ : Health issues for adolescents. Paediatr Child Health 2007; 17(11): 433-8.

24.- Turkel S, Pao M: Late consequences of pediatric chronic illness. Psychiatr Clin North Am 2007; 30 (4): 819-35.

25.- Corona F, Peralta E: Prevención de conductas de riesgo. Rev Med Clin Condes 2011; 22 (1): 68-75.

26.- Breinbauer $C$ : ¿Cómo construir un programa en prevención de salud para adolescentes? Organización Panamericana de la Salud, 2003. http://www.paho.org/Spanish/ AD/FCH/CA/Construir.ppt, última visita 24-04-2011.

27.- Bethell C, Klein J, Peck C: Assessing health system provision of adolescent preventive services: The young adult health care survey. Med Care 2001; 39 (5): 47890 .

28.- Radezik M, Sherer S, Neinsten L: Common concerns of adolescents and their parents. En: Neinsten L, Gordon C, Katzman D, Rosen D, Woods E. Adolescent Health Care. A practical guide. Fifth Edition, Philadelphia: Wolters Kluwer Health/ Lippincott Williams \& Wilkins, 2008: 969-71.

29.- Vázquez S, Berner E, Calandra N: Estrategia para la reducción de oportunidades perdidas en salud reproduc- 
tiva. Arch Argent Pediatr 2003; 101 (6): 466-9.

30.- Fonseca H: Helping adolescents develop resilience: steps the pediatrician can take in the office. Adolesc Med State Art Rev 2010; 21 (1): 152-60, xi.

31.- Youngblade LM, Theokas C, Schulenberg J, Curry L, Huang IC, Novak M: Risk and promotive factors in families, schools, and communities: A contextual model of positive youth development in adolescence. Pediatrics 2007; 119 Suppl 1: S47-53.

32.- Duncan PM, García AC, Frankowski BL, et al: Inspiring healthy adolescent choices: a rationale for and guide to strength promotion in primary care. J Adolesc Health 2007; 41 (6): 525-35.

33.- Mohajer $N$, Earnest J: Widening the aim of health promotion to include the most disadvantaged: vulnerable adolescents and the social determinants of health. Health Educ Res 2010; 25 (3): 387-94.

34.- Perrin J M, Romm D, Bloom SR, et al: A family-centered, community-based system of services for children and youth with special health care needs. Arch Pediatr Adolesc Med 2007; 161 (10): 933-6.

35.- Maddaleno M, Morello P, Infante-Espinola F: Salud y desarrollo de adolescentes y jóvenes en Latinoamérica y El Caribe: desafíos para la próxima década. Salud Publica Mex 2003; 45 suppl 1: S132-9.

36.- Schor EL: Family pediatrics: report of the Task Force on the Family. Pediatrics 2003; 111 (6 Pt 2): 1541-71.

37.- Newman K, Harrison L, Dashiff C, Davies S: Relationships between parenting styles and risk behaviors in adolescent health: An integrative literature review. Rev Latino-am Enfermagem 2008; 16 (1): 142-50.

38.- Santander S, Zubarew T, Santelices L, Argollo P, Cerda $J$. Bórquez M: Influencia de la familia como factor protector de conductas de riesgo en escolares chilenos. Rev Med Chile 2008; 136: 317-24.

39.- Franck KL, Buehler C: A family process model of marital hostility, parental depressive affect, and early adolescent problem behavior: The roles of triangulation and parental warmth. J Fam Psychol 2007; 21(4): 61425.

40.- Cui M, Donnellan MB, Conger RD: Reciprocal influences between parents' marital problems and adolescent internalizing and externalizing behavior. Dev Psychol 2007; 43 (6): 1544-52.

41.- Buehler C: Parents and peers in relation to early adolescent problem behavior. J Marriage Fam 2006; 68: 109-24.

42.- Child Health and Maternity Services Branch, Department of Health, United Kingdom: Transition: getting it right for young people. Improving the tran- sition of young people with long term conditions from children's to adult health services. 2006.

43.- Blum R, Garell D, Hodgman C et al: Transition from child-centered to adult health-care systems for adolescents with chronic conditions. A position paper of the Society for Adolescent Medicine. J Adolesc Health 1993; 14 (7): 570-6.

44.- Jennison $M H$, Coleman AB, Feiertag RB, et al: Age limits of pediatrics. Pediatrics 1972; 49 (3): 463.

45.- American Academy of Pediatrics Council on Child and Adolescent Health: Age limits of pediatrics. Pediatrics 1988; 81 (5): 736.

46.- Litt IF: Age Limits of Pediatrics, American Academy of Pediatrics, Council on Child Health, Pediatrics, 1972; 49: 463. Pediatrics 1998; 102: 249-50.

47.- American Academy of Pediatrics: AAP Publications Retired and Reaffirmed. Pediatrics 2006; 117: 1846-7.

48.- Boston Medical Center: Pediatrics-Adolescent Center. http://www.bmc.org/pediatrics-adolescentcenter.htm, última visita 09-05-2011.

49.- Children's National Medical Center: Departments and Programs. Adolescent and Young Adult Medicine.http:// www.childrensnational.org/DepartmentsandPrograms/ default.aspx?Id=370\&Type=Dept\&Name=Adolesce nt\%20and\%20Young\%20Adult\%20Medicine, última visita 09-05-2011.

50.- Children's Hospital Los Angeles: Division of Adolescent Medicine. http://www.chla.org/site/pp.aspx?c=ipINKTO AJsG\&b=3750929, última visita 09-05-2011.

51.- Miami Children's Hospital: Medical Services. Adolescent Medicine. http://www.mch.com/page/EN/242/ Medical-Services/Adolescent-Medicine.aspx, última visita 09-05-2011.

52.- University of Minnesota: Adolescent Health and Medicine. Department of Pediatrics. http://www.med.umn. edu/peds/ahm/, última visita 09-05-2011.

53.- UCSF School of Medicine: Department of Pediatrics. Division of Adolescent Medicine. http://pediatrics. medschool.ucsf.edu/youth/, última visita 09-05-2011.

54.- Stanford School of Medicine: Pediatrics. Adolescent Medicine. http://adolescent.stanford.edu/, última visita 09-05-2011.

55.- Ministerio de Salud Pública: Dirección Nacional de Atención Materno Infantil y Planificación Familiar. Programa Nacional de Atención Integral a la Salud de Adolescentes, Cuba. Actas 22 $2^{\mathrm{a}}$ y $23^{\mathrm{a}}$ Reunión Comité de Adolescencia de ALAPE (Asociación Latinoamericana de Pediatría), Montevideo, Uruguay 2009 y Bahía, Brasil 2010.

56.- Ministério da Saúde, Secretaria de Atenção à Saúde, 
Departamento de Ações Programáticas Estratégicas: Diretrizes Nacionais para a Atenção Integral à Saúde de Adolescentes e Jovens na Promoção, Proteção e Recuperação da Saúde. Brasília, 2010.

57.- Lastra J: Estudio acerca del uso y la gestión de camas hospitalarias en la Región Metropolitana. http://www. saludyfuturo.cl/documentos/GESTIONCAMAS.pdf, última visita 08-05-2011.

58.- Facultad de Medicina, Universidad de Chile: Medichi: Red de Aprendizaje Digital en Salud. Diploma Salud Integral del Adolescente. http://www.medichi.cl/minisitios/salud_integral_adolescente/descripcion.htm, última visita 24-04-2011.

59.- UCvirtual: Diploma Desarrollo y Salud Integral del Adolescente 2010. http://www.uc.cl/ucvirtual/medicina/ adolescente2011/index.html, última visita 10-04-2011.

60.- Escuela de Postgrado, Facultad de Medicina, Uni- versidad de Chile: Programa de Título de Especialista Derivado de la Pediatría Adolescencia. Hospital Luis Calvo Mackenna, Centro Adolescencia "Ser Joven". http://www.postgradomedicina.uchile.cl/med.portal?_nfpb=true\&url=10127\&_pageLabel=conUrl\&1=1, última visita 24-04-2011.

61.- Fatusi AO, Hindin MJ: Adolescents and youth in developing countries: Health and development issues in context. J Adolesc 2010; 33(4): 499-508.

62.- A New Approach to Classifying Adolescent Developmental Stages. En: Breinbauer C, Maddaleno M. Youth: Choices and Change, Washington, D.C.: Pan American Health Organization. Scientific and Technical Publication No594, 2005: 257-68.

63.- Casey BJ, Duhoux S, Malter Cohen M: Adolescence: what do transmission, transition, and translation have to do with it? Neuron 2010; 67 (5): 749-60. 